\title{
LA TÉLÉMÉDECINE EN ZONES RURALES : REPRÉSENTATIONS ET EXPÉRIENCES DE MÉDECINS GÉNÉRALISTES
}

Maxime Durupt, Olivier Bouchy, Sonia Christophe, Joëlle Kivits, Jean-Marc Boivin

\author{
S.F.S.P. | « Santé Publique »
}

2016/4 Vol. 28 | pages 487 à 497

ISSN 0995-3914

Article disponible en ligne à l'adresse :

http://www.cairn.info/revue-sante-publique-2016-4-page-487.htm

\section{Pour citer cet article :}

Maxime Durupt et al., " La télémédecine en zones rurales : représentations et expériences de médecins généralistes », Santé Publique 2016/4 (Vol. 28), p. 487-497.

Distribution électronique Cairn.info pour S.F.S.P..

(C) S.F.S.P.. Tous droits réservés pour tous pays.

La reproduction ou représentation de cet article, notamment par photocopie, n'est autorisée que dans les limites des conditions générales d'utilisation du site ou, le cas échéant, des conditions générales de la licence souscrite par votre établissement. Toute autre reproduction ou représentation, en tout ou partie, sous quelque forme et de quelque manière que ce soit, est interdite sauf accord préalable et écrit de l'éditeur, en dehors des cas prévus par la législation en vigueur en France. Il est précisé que son stockage dans une base de données est également interdit. 


\section{La télémédecine en zones rurales: représentations et expériences de médecins généralistes Telemedicine in rural areas: general practitioners' representations
and experiences} Maxime Durupt ${ }^{1}$, Olivier Bouchy ${ }^{1}$, Sonia Christophe ${ }^{2}$, Joëlle Kivits ${ }^{1,3,4}$, Jean-Marc Boivin ${ }^{1}$

\section{$\hookrightarrow \rightarrow$ Résumé}

Introduction : La télémédecine constitue une nouvelle forme de pratique médicale dont le développement est aujourd'hui en pleine expansion. Elle trouve un écho particulier dans certains territoires lorrains où le déficit en médecins généralistes et spécialistes nécessite le développement de nouvelles formes de pratiques. L'objectif de cette étude était d'explorer les représentations de la télémédecine, et de la téléconsultation en particulier, des médecins généralistes exerçant en zones déficitaires en offre de soins en Lorraine et d'identifier les avantages et désavantages perçus de son développement.

Méthodes: Une analyse qualitative a été faite à partir de cinq focus groupes réalisés avec 32 médecins entre juin 2014 et juillet 2015 dans un territoire à faible densité médicale.

Résultats : Cette étude montre qu'il existe une méconnaissance générale de la télémédecine. Les médecins expriment leur désir de préserver leur rôle de pivot et d'être acteurs de cette télémédecine qui ne devra pas leur être imposée. Les réticences en termes d'aspects juridiques et financiers sont des freins à son développement. Enfin, la télémédecine devra respecter un cadre légal en termes de responsabilité médicale et de sécurisation des données.

Discussion : Chaque mois, plus de cent actes de télémédecine sont réalisés en Lorraine. Bien qu'il s'agisse d'une solution permettant de faciliter l'accès aux soins dans les zones déficitaires, les médecins semblent vouloir préserver leur relation médecin-patient et ne se sentent pas prêts à modifier leur pratique.

Mots-clés: Télémédecine ; Téléconsultation ; Médecine générale ; Démographie médicale ; Recherche qualitative.

\section{$\hookrightarrow \rightarrow$ Summary}

Introduction: Telemedicine is a rapidly growing new mode of healthcare practice. It is particularly used and needed in remote areas in Lorraine (North East of France) that currently face a shortage of general practitioners and specialists. The objective of this study was to analyse general practitioner's representations of telemedicine and teleconsultation. The study also identified the advantages and disadvantages of this new mode of medical practice.

Methods: A qualitative research was led: 5 focus groups were conducted with 32 doctors in areas faced with the problems of health professional shortages between June 2014 and July 2015. Results: This study reveals a general ignorance of telemedicine. Doctors want to play a central role in this new form of medical practice which must remain optional. Their reluctance essentially concerns financial and legal aspects that constitute obstacles to the development of telemedicine. Finally, this new mode of medical practice must comply with a legal framework regarding medical responsibility and personal data protection.

Discussion: More than 100 medical procedures are delivered by telemedicine in Lorraine each month. This new technology is a solution to provide healthcare services in medically underserved areas. However, general practitioners want to preserve the "doctor-patient" relationship and do not wish to change their practice.

Keywords: Telemedicine; Teleconsultation; General practice; Medical demography; Qualitative research.

\footnotetext{
${ }^{1}$ Département de Médecine générale de Nancy - Université de Lorraine - 5 avenue de la Forêt de Haye - 54505 Vandœuvre-lès-Nancy cedex.

${ }^{2}$ Doctorante - Laboratoire de Sociologie et d'Anthropologie - Université de France-Comté - France.

${ }^{3}$ Université de Lorraine - Université Paris Descartes - Apemac - EA4360 - Nancy F-54000 - France.

${ }^{4}$ CHU Nancy / Pôle QSP2 - Service Évaluation et Information Médicales - Nancy F-54000 - France.
} 


\section{Introduction}

Face à la faible démographie médicale que connaissent certaines régions françaises dont la Lorraine, la télémédecine est présentée comme une solution prometteuse [1]. Joindre à distance un professionnel permettrait de répondre plus rapidement aux besoins d'un patient présentant une maladie chronique ou une pathologie aiguë et ce dans le respect d'une prise en charge efficiente et de qualité. Au-delà de sa contribution à l'amélioration de la qualité de l'offre de soins, la télémédecine pourrait permettre également de répondre aux inégalités territoriales de santé [2]. Urbains, ruraux, semi-ruraux bénéficieraient ainsi d'une offre de soins équitable [3].

La télémédecine constitue une nouvelle forme de pratique médicale dont le développement est aujourd'hui en pleine expansion. En France, la loi « Hôpital, patient, santé, territoires » du 21 juillet 2009 dite loi HPST définit la télémédecine et lui donne un cadre légal. «La télémédecine est une forme de pratique médicale à distance utilisant les technologies de l'information et de la communication » [4].

Le décret du 19 octobre 2010 relatif à la télémédecine en définit les cinq axes constitutifs [5] : la téléconsultation, la télé-expertise, la télésurveillance médicale, la téléassistance médicale, la réponse médicale ou régulation médicale.

Actuellement, une expérimentation menée dans cinq régions françaises (Aquitaine, Bourgogne, Île-de-France, Rhône-Alpes et Océan Indien) vise à améliorer le système de soins par le biais de l'usage du numérique. L'objectif est de généraliser les solutions qui auront montré leur efficacité et de fixer un cadre légal à la télémédecine. Bénéficiant d'un contexte national porteur, l'Agence régionale de santé $\left(\mathrm{ARS}^{1}\right)$ de Lorraine a initié plusieurs projets sur son territoire présentés dans son «Programme régional de télémédecine » (PRT) [6]. Il prévoit des expérimentations dans les domaines de la psychiatrie, de l'addictologie, de la chirurgie et même de la génétique en plus des domaines prioritaires fixés au niveau national par la Direction générale de l'offre de soins $\left(\right.$ DGOS$^{2}$ ) (permanence des soins en imagerie, prise en charge de l'accident vasculaire cérébral (AVC), etc.).

La télémédecine trouve un écho particulier en Lorraine. Ce territoire fait face à une problématique de démographie

\footnotetext{
${ }^{1}$ ARS : établissement public administratif chargé de la mise en œuvre de la politique de santé dans sa région.

${ }^{2}$ DGOS : Dépend du ministère de la santé, a pour mission d'organiser l'offre de soins.
}

médicale, particulièrement en Meuse et dans le sud-toulois. Le déficit en médecins généralistes et spécialistes nécessite le développement de nouvelles formes de pratiques. La télémédecine porte l'espoir d'une meilleure couverture des zones éloignées en offre de soins de second recours. Or, les expérimentations régionales demeurent essentiellement portées sur des soins aigus, en lien avec une prise en charge hospitalière ou des soins spécialisés. Ainsi, dans la plupart de ces cas, le médecin généraliste ne joue pas un rôle prépondérant. À notre connaissance, peu d'études se sont intéressées aux représentations de la télémédecine par les médecins généralistes français [7, 8]. À l'étranger, la télémédecine est bien accueillie par les paramédicaux mais les médecins généralistes expriment majoritairement leurs réticences face à cette technologie $[9,10]$. Cette dimension est cependant essentielle à explorer car toute innovation ne peut exister que par le sens social que leur donneront les usagers [11]. La France a pris du retard par rapport aux pays anglo-saxons et scandinaves, où les téléconsultations font déjà partie de leur pratique depuis une vingtaine d'années. [12]

Dans ce contexte nous nous sommes intéressés à la perception des médecins généralistes exerçant en zones déficitaires en offre de soins en Lorraine, vis-à-vis de la télémédecine. Quelles étaient leurs attentes face à ces dispositifs innovants, particulièrement au sein des territoires ruraux ? L'objectif était d'explorer les représentations qu'ils se faisaient de la télémédecine, et de la téléconsultation en particulier. Nous avons cherché à identifier les avantages et désavantages perçus de son développement.

\section{Méthodes}

Une étude qualitative par focus groupes a été réalisée. Cette méthode a été choisie pour sa capacité à produire, dans des délais courts, une grande variété d'idées, d'opinions et de croyances existant sur un sujet donné [13]. Les caractéristiques de ce travail ont été développées selon les critères COREQ [14].

Cinq focus groupes ont été réunis entre juin 2014 et juillet 2015 : quatre dans la Meuse et un dans le sud-toulois (figure 1). Ces territoires ont été choisis en raison de leur faible densité médicale et de leur éloignement par rapport au Centre hospitalier de Nancy.

Les participants de l'étude étaient des médecins généralistes installés en libéral conventionné en secteur 1 exerçant 


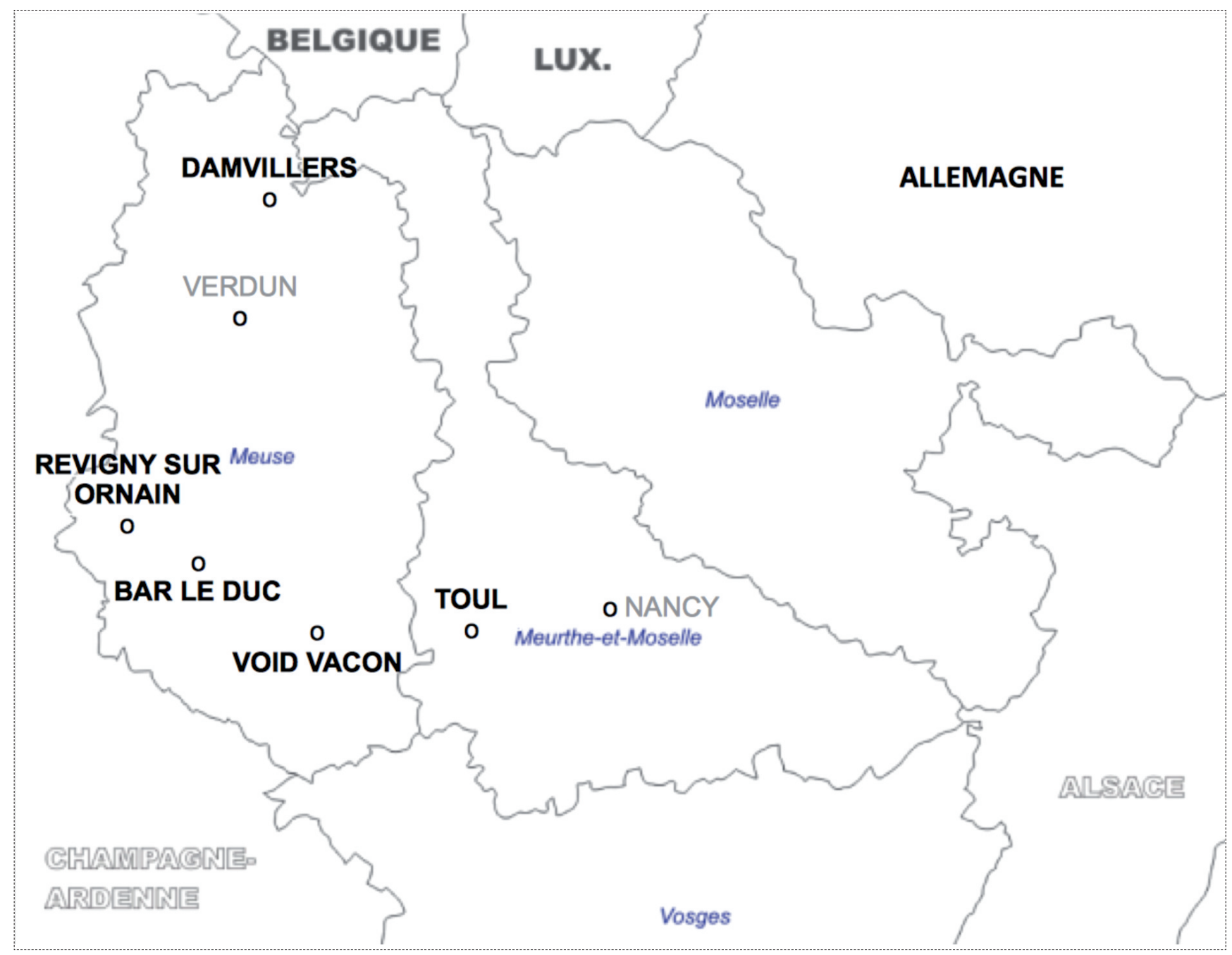

Figure 1 : Carte représentant les cinq lieux de réunions: Revigny-sur-Ornain, Bar-le-Duc, Toul, Void-Vacon pour le groupe Commercy, Damvillers pour le groupe Verdun

sur le territoire concerné par l'étude. Le recrutement s'est fait par téléphone, par échantillonnage de commodité en recherche de variation maximale. Les médecins étaient répartis dans les différents groupes en fonction de leur emplacement géographique. Au total, 32 médecins ont participé à l'étude. Les entretiens ont duré en moyenne 62 minutes.

Un guide d'entretien a été élaboré et testé par une étude pilote. Il comprenait cinq questions ouvertes et des questions de relances afin d'enrichir le débat.

Dans un premier temps, la discussion s'ouvrait sur la télémédecine en général afin d'en recueillir la perception de chacun. Puis, le champ du débat était recentré sur la téléconsultation en particulier.
L'auteur (MD) était présent lors des cinq entretiens. Il était animateur pour deux d'entre eux, assisté par une sociologue (SC). Il était observateur dans les trois autres groupes, animés par un médecin généraliste (OB).

Les entretiens ont été enregistrés après information et accord oral des participants. Ils ont été ensuite intégralement retranscrits en assurant l'anonymat des participants. Les entretiens ont été analysés thématiquement [15] : les thèmes abordés, à partir du guide d'entretien et ceux émergeant spontanément lors des focus groupes ont été identifiés. Ils ont ensuite été organisés en unités de sens et catégorisés afin de répondre à la question guidant la recherche. L’analyse a été réalisée individuellement par MD, $\mathrm{JK}$ et $\mathrm{OB}$. 


\section{Résultats}

\section{Description de la population (tableau I)}

La moyenne d'âge des participants était de 51,5 ans [ 31 ; 67]. Un tiers des participants étaient des femmes. Les trois quarts d'entre eux travaillaient en cabinet de groupe ${ }^{3}$; $53 \%$ en zone rurale, $34 \%$ en zone semi-rurale et $13 \%$ en zone urbaine. Tous les médecins étaient informatisés.

${ }^{3}$ Cabinet de groupe: cabinet médical regroupant au moins deux médecins.
Aucun participant n'avait d'expérience concernant la pratique de la télémédecine.

La distance moyenne de leur cabinet à l'hôpital le plus proche était de $21 \mathrm{~km}$. La distance moyenne avec le Centre hospitalier universitaire de Nancy était de $74 \mathrm{~km}$. Un tiers des participants avaient moins de 15 ans d'installation, un tiers entre 15 et 30 ans et un tiers plus de 30 ans.

\section{Représentations de la télémédecine (tableau II)}

\section{Une définition imprécise}

Les connaissances qu'ont les médecins généralistes de la télémédecine sont très variables. Leur difficulté à

Tableau I : Population de l'étude

\begin{tabular}{|c|c|c|c|c|}
\hline Médecins & Âge (années) & Sexe & Exercice & Milieu \\
\hline \multicolumn{5}{|c|}{ Revigny-sur-Ornain (55) } \\
\hline$-\mathrm{R} 1$ & 64 & $\mathrm{H}$ & Groupe & Semi-rural \\
\hline$-\mathrm{R} 2$ & 67 & $\mathrm{H}$ & Groupe & Semi-rural \\
\hline$-\mathrm{R3}$ & 53 & $\mathrm{~F}$ & Groupe & Semi-rural \\
\hline$-\mathrm{R} 4$ & 55 & $\mathrm{H}$ & Groupe & Semi-rural \\
\hline \multicolumn{5}{|l|}{ Bar-le-Duc (55) } \\
\hline$-B 1$ & 62 & $\mathrm{H}$ & Seul & Rural \\
\hline$-B 2$ & 37 & $\mathrm{H}$ & Groupe & Rural \\
\hline$-\mathrm{B} 3$ & 57 & $\mathrm{H}$ & Groupe & Rural \\
\hline$-B 4$ & 59 & $\mathrm{H}$ & Groupe & Urbain \\
\hline$-B 5$ & 50 & $\mathrm{H}$ & Groupe & Rural \\
\hline$-\mathrm{B} 6$ & 59 & $\mathrm{H}$ & Seul & Urbain \\
\hline$-B 7$ & 59 & $\mathrm{H}$ & Groupe & Semi-rural \\
\hline$-\mathrm{B} 8$ & 61 & $\mathrm{H}$ & Groupe & Rural \\
\hline$-B 9$ & 31 & $\mathrm{~F}$ & Groupe & Rural \\
\hline \multicolumn{5}{|l|}{ Commercy (55) } \\
\hline$-\mathrm{Cl}$ & 62 & $\mathrm{H}$ & Groupe & Semi-rural \\
\hline$-C 2$ & 32 & $\mathrm{H}$ & Groupe & Semi-rural \\
\hline$-c 3$ & 53 & $\mathrm{H}$ & Groupe & Rural \\
\hline$-\mathrm{C} 4$ & 51 & $\mathrm{H}$ & Groupe & Rural \\
\hline$-\mathrm{C5}$ & 60 & $\mathrm{~F}$ & Seul & Semi-rural \\
\hline \multicolumn{5}{|l|}{ Verdun (55) } \\
\hline$-\mathrm{V} 1$ & 49 & $\mathrm{~F}$ & Seul & Semi-rural \\
\hline$-\mathrm{V} 2$ & 36 & $\mathrm{~F}$ & Groupe & Rural \\
\hline$-\mathrm{V} 3$ & 51 & $\mathrm{H}$ & Groupe & Rural \\
\hline$-\mathrm{V} 4$ & 43 & $\mathrm{H}$ & Seul & Rural \\
\hline$-\mathrm{V} 5$ & 57 & $\mathrm{H}$ & Groupe & Rural \\
\hline$-\mathrm{V} 6$ & 50 & $\mathrm{H}$ & Groupe & Semi-rural \\
\hline \multicolumn{5}{|l|}{ Toul (54) } \\
\hline$-\mathrm{T} 1$ & 31 & $\mathrm{~F}$ & Groupe & Rural \\
\hline$-\mathrm{T} 2$ & 60 & $\mathrm{H}$ & Groupe & Rural \\
\hline$-\mathrm{T} 3$ & 33 & $\mathrm{H}$ & Groupe & Urbain \\
\hline$-\mathrm{T} 4$ & 58 & $\mathrm{H}$ & Groupe & Rural \\
\hline$-\mathrm{T} 5$ & 62 & $\mathrm{H}$ & Seul & Semi-rural \\
\hline$-\mathrm{T} 6$ & 38 & $\mathrm{H}$ & Groupe & Rural \\
\hline$-\mathrm{T} 7$ & 47 & $\mathrm{~F}$ & Seul & Urbain \\
\hline$-\mathrm{T} 8$ & 60 & $\mathrm{~F}$ & Seul & Rural \\
\hline
\end{tabular}


fournir une définition met en évidence une méconnaissance et des projections approximatives de cette forme de pratique médicale : "je ne sais pas s'il y a une définition précise » (T4), «ça reste quelque chose de très abstrait pour l'instant» (V3).

Paradoxalement, les médecins parviennent à évoquer les différents axes de la télémédecine. Pour la téléconsultation, il est question «de cabines de téléconsultation» (B5). La télésurveillance médicale est définie comme «le suivi de paramètres qui peuvent être enregistrés à la maison» (C4). Pour la télé-expertise, ils évoquent « ce qui se fait déjà dans les réunions de concertation pluridisciplinaires » (R2). La téléassistance médicale est évoquée au travers de l'exemple des «chirurgies à distance, avec des robots» (V1). Enfin, la réponse médicale, lorsqu'ils sont «guidés par les médecins du SAMU» (C3).

Concernant la téléconsultation, deux éléments sont essentiels à leur compréhension. D'une part, elle est perçue comme une aide à distance permettant de contacter un professionnel de santé pour un avis : "on suppose qu'on peut communiquer visuellement avec un professeur ou une personne compétente en dermatologie, en neurologie pour nous aider » (R1). Plus qu'une innovation, elle est perçue comme un prolongement des pratiques existantes. Un médecin parle d'une "ébauche avec le téléphone depuis longtemps déjà » (V6).

D'autre part, la téléconsultation est décrite comme un moyen technique favorisant l'accès aux soins et permettant de «rapprocher la campagne de la ville» (R1). Les médecins voient la possibilité de s'affranchir des distances "pour les patients qui ne sont pas faciles à transporter» (B4) et ainsi leur permettre « d'épargner le temps de transport» (B2).

\section{Une opportunité pour innover sa pratique}

L'intérêt de ces nouveaux modes d'exercice se situe, pour les médecins rencontrés, dans le contexte contraint actuel de l'offre de soins : « on est ralenti quand on veut des rendezvous. C'est bien simple, un rendez-vous maintenant c'est six mois » (R1). La télémédecine apparaît alors comme une solution à envisager, étant donné le faible nombre de spécialistes dans certains territoires.

La téléconsultation apparaît aussi comme une forme de pratique médicale dont l'intérêt reste à démontrer, les technologies actuelles satisfaisant déjà les praticiens : « On a fait comme ça jusqu'à maintenant. Pourquoi est-ce qu'on changerait» (V6). Cependant son développement leur paraît cohérent dans la continuité de l'informatisation des cabinets : «c'est inéluctable, comme l'informatisation» (B8).
Tableau II : Représentations de la télémédecine

\begin{tabular}{l}
\multicolumn{1}{c}{ Représentations de la télémédecine } \\
\hline \multicolumn{1}{c}{ Aspects positifs } \\
\hline - Facilite l'accès aux soins \\
- Déjà pratiquée \\
- Évolution logique de la pratique médicale \\
- Engouement envers les nouvelles technologies \\
\hline \multicolumn{1}{c}{ Aspects négatifs } \\
\hline - Méconnaissance de la télémédecine et de ses applications \\
- Peu de spécialités de second recours envisagées \\
- Perte de l'examen clinique physique \\
- Pégradation de la relation médecin-patient
\end{tabular}

\section{Les spécialités envisagées en téléconsultation}

Selon les médecins rencontrés, peu de spécialités répondent à la nécessité d'une téléconsultation.

La dermatologie est le recours le plus abordé et paraît la mieux adaptée car « si on peut montrer par télétransmission la lésion cutanée, ça pourrait être intéressant» (R2). «La dermatologie, ça me paraît quelque chose d'évident» (C4).

La neurologie est la deuxième spécialité abordée : «la neurologie, c'est (la spécialité) la plus dure d'accès » (V6), puis la psychiatrie "tout ce qui est dépression, addiction» (V1).

D'autres domaines sont, au contraire, jugés inadaptés car la difficulté réside, selon eux, dans les gestes techniques qui ne sont pas réalisables à distance : «La consultation de télémédecine ne va pas supprimer l'acte technique du spécialiste» (R1).

\section{Préserver la place du patient et la qualité} de la relation médecin-patient

La télémédecine est aussi envisagée du point de vue de la consultation et des transformations potentielles sur la relation entre le médecin et le patient. Le contact physique est au cœur des discussions, la télémédecine représentant une menace à la préservation de ce lien essentiel à l'examen clinique, au bon diagnostic et à l'aspect relationnel caractéristique de leur pratique de médecine générale: «il ne faut pas shunter l'examen clinique du médecin » (T3). Pour certains participants, il s'agit d'une vraie limite au développement de la télémédecine : "Le contact ne sera jamais remplacé par une machine » (T1).

Les dispositifs de télémédecine sont par ailleurs perçus comme générant une perte d'information essentielle venant 
de la rencontre en face-à-face avec le médecin. Téléconsulter supprime la première phase de consultation qui commence dès la porte du cabinet franchie : «il manque quand même toute la partie où le patient rentre dans le bureau et où on commence déjà à se dire il y a ci, il y a ça» (V4), « il y a un risque majeur de sous diagnostic ou de perte d'information » (B5).

Les participants expriment aussi leurs craintes vis-à-vis de la demande des patients qui, selon l'avis général, «vont être demandeurs » (B9). Le caractère novateur et immédiat risque de prendre le pas sur l'écoute au cœur du colloque singulier: "c'est nouveau, c'est moderne» (V6), il y a un "besoin d'immédiateté " (V1). Les demandes abusives de la part des patients auxquelles devront faire face les praticiens sont craintes : "si c'est eux qui peuvent avoir accès à la télémédecine, il y aura des débordements, ils appelleront pour tout et n'importe quoi, ce sera l'anarchie la plus totale » (T8).

Enfin, les médecins évoquent le risque de «dépersonnaliser » (T1) le patient : "si c'est un discours entre experts et qu'on ne parle plus qu'entre nous» (T1) et le risque d'un « désengagement du médecin vis-à-vis de ses patients » (V1).

\section{L'organisation d'une téléconsultation (tableau III)}

Une mise en cuvre complexe, orchestrée par le médecin généraliste

Pratiquer la télémédecine apparait complexe pour les médecins rencontrés. Les réticences sont nombreuses, à la fois techniques, logistiques ou relatives à la formation. En l'absence d'exemples concrets de proximité, les médecins développent des représentations négatives quant à l'organisation de la télémédecine et posent alors des conditions nécessaires à son bon développement : «il faut que ce soit organisé. Il faut un cadre » (C5).

Le médecin généraliste doit rester, selon eux, le « pivot » de la téléconsultation. En effet, tout dispositif de télémédecine doit s'inscrire dans un parcours de soins ${ }^{4}$ cohérent où le médecin généraliste occupe une position centrale qu'il convient de conserver : "le médecin traitant doit garder son rôle, son utilité pour se faire l'interprète de son patient» (R2) afin "d'avoir des vrais échanges et de rester en position de coordination " (T1), "c'est nous qui allons poser l'indication » (C4).

\footnotetext{
${ }^{4}$ Le parcours de soins consiste à confier au médecin traitant la coordination des soins pour le suivi médical du patient.
}

L'inscription de la télémédecine dans le parcours de soins du patient est une garantie pour le médecin qui reste maître de sa pratique, mais surtout une condition sine qua non à son utilisation : "si ce n'est pas un outil que moi j'ai choisi, il risque d'y avoir de la poussière dessus » (T4). La crainte est de se voir imposer des manières de pratiquer qui ne leur correspondent pas, par des instances publiques, des sociétés privées ou des mutuelles: "sinon ça va être des grandes boites qui vont nous proposer des systèmes clé en main et on aura pas du tout ce qu'on veut» (T8).

La formation des médecins est enfin évoquée car «il faudra un temps d'initiation » (C1), temps nécessairement long et complexe à mettre en place, sans compter le temps d'apprentissage du côté du patient.

\section{Gain ou perte de temps?}

La télémédecine est tantôt vue comme un atout, tantôt comme un obstacle dans l'organisation de leur «temps médical » (B2). L'atout est de pouvoir faire gagner du temps au patient, notamment dans l'accès plus rapide à un spécialiste : "on passe aujourd'hui pratiquement plus de temps à relancer les spécialistes pour obtenir un rendezvous qu'à faire avancer le patient ». L'obstacle est la perte de temps, la téléconsultation étant considérée comme «chronophage» (R2) : « on va passer deux ou trois fois plus de temps pour faire cette téléconsultation » (T4). Les participants estiment ainsi que la télémédecine ne doit pas se généraliser et remplacer les pratiques habituelles, mais qu'elle devra rester « une activité annexe, partielle» (V4), utilisée " une fois de temps en temps " (R1).

Par ailleurs, les disponibilités des médecins spécialistes étant déjà limitées en consultation physique, il leur est difficile d'imaginer que les médecins experts "se rendent suffisamment disponibles pour apparaître à l'écran immédiatement» (V6).

Tableau III : Organisation d'une téléconsultation

\begin{tabular}{l} 
Organisation d'une téléconsultation \\
\hline \multicolumn{1}{c}{ Souhaits des médecins } \\
\hline - Être le pivot de la téléconsultation \\
- À développer avec des partenaires locaux, \\
dans des lieux de proximité \\
- Accompagnement du patient \\
\hline \multicolumn{1}{c}{ Craintes } \\
\hline - Complexité \\
- Formation des médecins, des patients \\
\hline
\end{tabular}




\section{Travailler avec des partenaires de proximité}

L'organisation d'une téléconsultation implique la présence physique ou distante, synchrone ou asynchrone de nouveaux partenaires, notamment l'expert télé-consulté qui ne sera pas nécessairement connu par les praticiens requérants et qui fait craindre une prise en charge de mauvaise qualité. Les médecins suggèrent alors qu'ils seraient plus en confiance avec des partenaires locaux : « il faudrait vraiment que je connaisse bien le correspondant en face» (B6).

Les patients et les paramédicaux sont identifiés comme les autres partenaires de la téléconsultation, bien que les médecins expriment des difficultés à imaginer les acteurs présents lors d'une consultation à distance. La présence du patient est elle-même remise en question: "le patient doit-il être présent ou pas, je ne sais pas. Dans certaines mesures oui » (R3) ; pour d'autres en revanche «il paraît indispensable que le patient soit là » (R2).

Conformément à leur souhait de préserver la corporéité de la relation médecin-patient, la majorité se voit aux côtés du patient, le spécialiste de l'autre côté : «je me vois plus avec mon patient dans mon cabinet et le spécialiste sur l'écran, mais c'est moi qui toucherai. Je me vois plus être acteur » (T7). Ce principe de présence n'est pas partagé par tous et deviendrait même une contrainte : «moi je n'assiste pas aux consultations quand j'envoie chez un spécialiste» (B6).

La présence des paramédicaux est enfin envisagée afin d'aider certains patients ou pour prendre un avis directement : "l'infirmière pourrait très bien avoir un contact avec le spécialiste» (R4).

Concernant le lieu d'exercice d'un acte de téléconsultation, c'est aussi la proximité qui prédomine : «il serait plus raisonnable de le faire à partir d'un cabinet de groupe ou d'une Maison de santé pluri-professionnelle (MSP) ${ }^{5}$ (R2) ou d'un Établissement d'hébergement pour personnes âgées dépendantes (EHPAD)» (V2).

\section{Aspects financiers et juridiques (tableau IV)}

\section{Aspects financiers}

La question polémique est celle de la rémunération d'un acte de téléconsultation : « comment je vais me faire payer? » (B1). Les médecins rencontrés ne sont pas sans idée sur ce

${ }^{5}$ MSP : Lieu regroupant des professionnels médicaux et paramédicaux permettant d'apporter des soins coordonnés de premier recours. sujet : "créer un nouvel acte, une nouvelle cotation» (B7), "s'acheminer vers une rémunération forfaitaire de l'acte» (R2).

Il s'agit également du coût du matériel jugé excessif : « $s i$ c'est estampillé matériel médical, ça va être très cher »(V5). Le financement "paraît compliqué» (R3) et les médecins rencontrés espèrent «qu'il ne sera pas à la charge des médecins» (V2).

Enfin, ils redoutent que la télémédecine soit avant tout un enjeu économique : "l'ARS met des sous là-dedans parce que ça rapporte plus » (B5). Il est supposé que le coût d'une téléconsultation sera moindre qu'un déplacement du patient. Le but de la télémédecine leur paraît relever avant tout d'une «économie de santé » (R3).

\section{Aspects juridiques}

Les médecins sont inquiets de l'absence de cadre légal à l'heure actuelle : " je ne sais pas à ce jour s'il existe une charte de la télémédecine» (T2). Ils ne connaissent pas « l'instance qui va décider de tout ça »(V6). Pour certains, il s'agira de l'ARS : «il y a aura des budgets donc ça va être défini par l'ARS» (V1) ; pour d'autres, les sociétés privées ou les mutuelles pourraient jouer un rôle : «tu auras une boîte derrière qui chapeautera le truc »(V4).

Le problème de la responsabilité est aussi évoqué et pour eux « il existera tel qu'il existe déjà aujourd'hui, chacun va endosser la responsabilité »(R2), « elle sera partagée?» (V2).

Se pose aussi la question de «la sécurité, de la confidentialité » (C4) des données afin d'éviter le risque "d'être piraté» (C1). Il faut préserver le «secret médical» (T1).

D'autre part, « il faudra l'accord du patient aussi. Avec une signature » (T5) car « il y a le droit à l'image» (T6) du patient.

Tableau IV : Aspects financiers et juridiques

\begin{tabular}{l} 
Aspects financiers et juridiques \\
\hline \multicolumn{1}{c}{ Souhaits des médecins } \\
\hline - Clarifier le cadre légal de la pratique de la téléconsultation \\
- Obtenir un financement du matériel par les instances \\
publiques \\
- Garantir une responsabilité médicale partagée \\
- Préserver le secret médical \\
\hline Craintes \\
\hline - Coût d'investissement excessif \\
- Mainmise des agences régionales de santé, des assurances \\
et des mutuelles
\end{tabular}




\section{Discussion}

Le débat sur la télémédecine intervient dans un contexte de changement des pratiques médicales. Le « pacte territoire-santé6 $»$ mis en place depuis 2012 [16] prévoit le déploiement de la télémédecine. Le projet de loi de modernisation du système de santé adopté en décembre 2015 met l'accent sur les soins de proximité. Ils permettront d'apporter une réponse au virage ambulatoire souhaité par les médecins [17]. De plus, on observe un accueil favorable de la part des patients envers les nouvelles technologies. Un sondage réalisé en 2013 révélait que $64 \%$ des 50-65 ans seraient intéressés par la téléconsultation [18]. Une étude récente révèle que les patients des zones isolées en Lorraine voient positivement le développement de la télémédecine, notamment en termes de gain de temps et de suivi des maladies chroniques. Les réticences des patients concernent essentiellement les craintes quant à la qualité des soins [19]. Cependant, l'enjeu est de taille car seuls $15 \%$ du territoire meusien sont éligibles à l'internet à très haut débit [20]. Ceci met à mal la fiabilité et la sécurisation de la connexion.

Les médecins interrogés citent peu d'exemples existants de téléconsultations, alors que la région Lorraine compte déjà plus d'une centaine d'actes de téléconsultation par mois [21] : la prise en charge des accidents vasculaires cérébraux dans les hôpitaux de Bar-le-Duc et Verdun, le centre de dialyse de Bar-le-Duc [22], les téléconsultations d'addictologie organisées dans la maison médicale de Revigny-sur-Ornain [23].

En outre, les médecins interrogés confondent de manière répétée téléconsultation et télé-expertise. En effet, ils évoquent rarement la possibilité de consulter eux-mêmes un patient à distance. Ils entrevoient plutôt la téléconsultation comme la possibilité d'obtenir un avis spécialisé rapide, parfois même sans la présence du patient.

Cette méconnaissance peut expliquer la difficulté qu'avaient les médecins rencontrés à se sentir concernés par cette nouvelle forme de consultation. Ce sentiment peut s'expliquer par le manque d'informations disponibles sur le sujet en termes d'expérimentations ou de formations ou par simple refus de l'innovation. En effet, $33 \%$ des médecins étaient installés depuis plus de 30 ans et ne souhaitaient pas voir évoluer leur mode d'exercice.

\footnotetext{
${ }^{6}$ Pacte territoire santé : programme du ministère de la santé visant à permettre l'accès à tous les Français à des soins de qualité.
}

De plus les médecins exerçant seuls ou en ville, expriment majoritairement un sentiment de réticence vis-à-vis de la télémédecine. On peut alors supposer que l'exercice seul ne permet pas l'organisation de consultations à distance puisque cela requiert un investissement de temps et d'argent trop important pour un seul médecin. La tendance actuelle est au regroupement des médecins en cabinet de groupe ou Maison de santé pluri-professionnelle [24] qui facilitent l'accès à de nouvelles formes de pratique médicale. Le phénomène d'émulation de groupe permet d'envisager de changer son mode d'exercice et d'accueillir des projets comme la téléconsultation.

Cette réticence n'est certes pas propre à la région. Manque de temps, dégradation de la relation médecin-patient, perte de l'examen clinique, risque d'erreur diagnostique sont les freins à la téléconsultation évoqués par des médecins généralistes interrogés dans une étude similaire menée dans les Pays de la Loire [25]. En Angleterre, une étude révèle également la crainte d'un surcroît de travail et d'une perte d'autonomie des médecins [10].

La télémédecine trouve un accueil favorable lorsque l'imagerie est au cœur du dispositif. Ainsi, alors que les participants mentionnent peu de spécialités pouvant être compatibles avec une téléconsultation, la dermatologie semble, elle, évidente : la visualisation y est primordiale et l'envoi de photographies par Internet ou smartphone est d'ores-et-déjà une pratique répandue [26]. Les expérimentations de télémédecine prometteuses, telles que les téléconsultations en EHPAD, ne sont pas ou peu mentionnées, alors qu'elles se développent actuellement sur le territoire français [27].

Selon Mathieu-Fritz et Esterle [28], «La culture professionnelle constitue dans certains cas un facteur d'opposition à l'usage du dispositif de télé-présence au sein du monde médical». Deux enjeux se nouent plus spécifiquement autour du développement de la télémédecine et peuvent expliquer les représentations négatives observées dans notre étude.

D'une part, la ritualisation de l'acte médical incarnée par la rencontre physique entre le médecin et son patient, et la nécessité d'entretenir un colloque singulier, intime et confidentiel, constituent des freins certains au développement de la télémédecine et à son appropriation par les professionnels. Les médecins expriment la crainte d'une dégradation de la relation médecin-patient par le biais de l'utilisation excessive de nouvelles technologies. L'interaction est primordiale en médecine générale [29] et les médecins rencontrés ne voient pas comment cela pourra être préservé en télémédecine. Ce sentiment de déshumanisation se retrouve également dans une étude 
réalisée auprès de médecins généralistes de FrancheComté [8].

D'autre part, l'utilisation de la téléconsultation suggère que l'on puisse faire appel à un médecin distant voire étranger. Ce partage des tâches entraîne la «délégation d'une partie du jugement » [28] du spécialiste vers un autre professionnel; or le jugement constitue l'essence même de l'identité professionnelle, particulièrement exacerbée dans le champ médical où la définition de la maladie est source de pouvoir [30]. Cette hypothèse génère la crainte d'une prise en charge de mauvaise qualité ou d'une médecine au rabais. Les plateformes téléphoniques de certaines grandes enseignes délocalisées à l'étranger contribuent à renforcer ce sentiment.

Mais les médecins évoquent aussi la crainte d'une perte d'information, la téléconsultation ne permettant pas de visualiser les étapes pré- et post- consultation. Évaluer le comportement du patient auprès des autres personnes présentes dans le cabinet, analyser sa démarche depuis la salle d'attente, son déshabillage, sont des étapes qui constituent l'essence de la pratique de la médecine générale : les évincer revient à dégrader la qualité du diagnostic, mais aussi de la prise en charge.

Les principaux avantages attribués à la télémédecine sont la facilité d'accès au second recours et la réduction des déplacements. En effet, la pénurie de médecins spécialistes de second recours dans ces territoires, libéraux ou hospitaliers, contraint les généralistes à adresser leurs patients vers les $\mathrm{CHU}$ voisins distants d'une centaine de kilomètres. Ainsi, les délais de rendez-vous sont longs et le déplacement des patients paraît inévitable. On observe d'ailleurs depuis 2007 une diminution de la densité de médecins généralistes et autres spécialités en Meuse, un vieillissement de la population et une augmentation du nombre de maladies chroniques [3]. La télémédecine serait donc une solution à la perte de chance due à cette pénurie de médecins.

De cette étude, apparaît clairement le souhait que le médecin traitant conserve son rôle de pivot. Si le développement de la téléconsultation peut sous-entendre un accès illimité du patient à toutes les spécialités, le médecin traitant doit rester au centre du parcours de soins coordonnés tel que le conçoit la loi du 13 août 2004, relative à l'assurance maladie [31].

De plus, les médecins interrogés ne souhaitent pas voir se reproduire le même schéma que celui qui a conduit à la généralisation du tiers-payant. Cette décision prise sans concertation avec les représentants des médecins est vécue comme une contrainte et est donc fortement contestée. Un scénario similaire s'était produit lors de l'avènement de la télétransmission des feuilles de soins. À l'époque, la mise en place du programme SESAM-vitale ${ }^{7}$ avait été décriée [32]. Les médecins ne veulent donc pas que la télémédecine leur soit imposée.

Cet outil doit surtout être conçu par et pour ses utilisateurs. Il semble en effet essentiel de questionner les usagers, comme dans tout cadre d'innovation technologique [11]. Les médecins veulent être acteurs de l'avènement de la téléconsultation. C'est dans cette optique que le Conseil National de l'Ordre des Médecins a publié ses préconisations dans son livre blanc sur la télémédecine [33]. De plus, on assiste à une montée en puissance du télé-conseil médical personnalisé. Cette "ubérisation » de la santé échappe aux réglementations concernant la télémédecine [34]. On craint un accès low cost à des plateformes étrangères ayant un code pénal ou civil différents. Sur ce point, l'Ordre des médecins propose plusieurs solutions telles que l'inscription de l'acte de téléconsultation dans la classification commune des actes médicaux ou la simplification de la réglementation de la télémédecine [35].

Concernant la responsabilité médicale en téléconsultation, elle est la même que pour un acte physique à la différence que le médecin requérant doit recueillir le consentement libre et éclairé du patient après information écrite ou orale. Une clarification sur ce point semble donc indispensable pour obtenir l'adhésion des médecins à cette forme de pratique médicale comme le précise le Dr Pierre Simon, alors Président de la Société française de télémédecine (SFT-Antel) [36].

À ce jour, peu de médecins sont formés à la télémédecine. Cependant des formations existent et sont validées par l'Organisme gestionnaire du développement professionnel continu (OGDPC) et sont dispensées, entre autres, par la Société française de télémédecine (SFT-Antel) [37, 38].

L'étude présente des limites car les focus groupes ont été animés par des médecins généralistes, ce qui a pu influencer les réponses des participants et créer un biais d'investigation. La co-animation a permis cependant de limiter ce risque, la deuxième personne modérant la conversation et veillant au respect du guide d'entretien.

Le premier focus groupe s'est déroulé au sein de la maison médicale où travaille l'un des co-auteurs de cet article. Cependant, ce dernier n'a pas participé à cette réunion afin de ne pas influencer ses collègues. Ce focus groupe était notre étude-pilote et les données recueillies ont été exploitées.

${ }^{7}$ SESAM-vitale : Système de communication informatique permettant la télétransmission de feuilles de soins électroniques. 
Nous avons réparti les focus groupes sur l'ensemble du territoire retenu en tentant d'avoir la plus grande variabilité de participants. Des médecins contactés ont refusé de participer par manque de temps ou crainte de non-respect de l'anonymat. D'autres ayant accepté de participer ne sont pas venus. Nous aurions pu réaliser des entretiens individuels afin de recueillir leur opinion.

\section{Conclusion}

Les médecins interrogés semblent démunis et expriment en majorité leurs réticences vis-à-vis de la télémédecine. Aujourd'hui, le numérique fait partie intégrante de la pratique médicale quotidienne. Les patients sont de plus en plus acteurs de leur santé grâce aux informations médicales relayées par Internet. En définitive, c'est la relation médecin-patient qui évolue. Pour en préserver la qualité, les médecins devront intégrer ces nouvelles technologies en adaptant leur exercice quotidien afin de rester le pivot du parcours de soins.

Diffuser les souhaits des médecins généralistes vis-à-vis de la télémédecine aura un impact sur son développement. La consultation à distance provoquera nécessairement un bouleversement dans le parcours de soins. C'est un enjeu pour la restructuration du système de soins. Pour généraliser cette forme de pratique médicale, des formations adaptées aux problématiques locales devront être proposées et pourquoi pas, dans un avenir proche, être intégrées au cursus universitaire. Enfin, la télémédecine, en France, devra sortir du modèle expérimental.

\section{Aucun conflit d'intérêt déclaré}

\section{Références}

1. Barlet M, Fauvet L, Guillaumat-Tailliet F, Olier L. Quelles perspectives pour la démographie médicale? La France et ses régions. Paris: Institut national de la statistique et des études économiques, Références; 2010;65-77.

2. Kubiak Y. Le Pacte territoire santé, garant de qualité et d'accessibilité des soins. Paris: Institut national de la statistique et des études économiques, Dossier Lorraine numéro 1. mai 2015 ; Disponible sur : $<\mathrm{http}$ ://www.insee.fr/fr/insee_regions/lor/themes/inseedossier/lor_ind_01/lor_ind_01_fic5.pdf $>$.
3. Le Breton-Lerouvillois G, Rault J-F. Atlas de la démographie médicale en France [internet]. Conseil national de l'ordre des médecins; janvier 2015. Disponible sur: <https://www.conseil-national. medecin.fr/node/1607>.

4. République Française. Code de la santé publique. Article L6316-1 loi HPST. legifrance.gouv.fr. 2009.

5. République Française. Code de la santé publique. Décret $n^{\circ} 2010$ 1229 du 19 octobre 2010 - art. 1. legifrance.gouv.fr. 2010.

6. Agence régionale de santé de Lorraine. Programme Régional de déploiement de la Télémédecine. 2011. Disponible sur : <www.ars. lorraine.sante.fr/fileadmin/LORRAINE/ARS_LORRAINE/ ACTUALITES/PRS/Plan_Action_PRT_v20111216.pdf×.

7. Mathieu S. La téléconsultation : I'avis des médecins généralistes dans les Alpes Maritimes [Thèse d'exercice]. [France] : Université de Nice-Sophia Antipolis. Faculté de Médecine ; 2012.

8. Lavaill L. Télémédecine : Définition, applications et enquête auprès des médecins généralistes de Franche-Comté.[Thèse d'exercice de médecine générale]. [Besançon] : Université de Franche-Comté, Faculté de médecine et de pharmacie ; 2011. Disponible sur : <www. sudoc.abes.fr.bases-doc.univ-lorraine.fr>.

9. Taylor J, Coates E, Brewster L, Mountain G, Wessels B, Hawley MS. Examining the use of telehealth in community nursing : identifying the factors affecting frontline staff acceptance and telehealth adoption. J Adv Nurs. 2015;71(2):326-37.

10. MacNeill V, Sanders C, Fitzpatrick R, Hendy J, Barlow J, Knapp M, et al. Experiences of front-line health professionals in the delivery of telehealth: a qualitative study. $\mathrm{Br} J$ Gen Pract. juillet 2014;64(624):e401-7.

11. Akrich $M$, Méadel C. Problématiser les usages. Sciences sociales et santé. 22(2):5-20.

12. Gagnon MP, Duplantie J, Fortin JP, Landry R. Implementing telehealth to support medical practice in rural/remote regions: what are the conditions for success? Implementation Science. 2006;1:18.

13. Kitzinger J, Markova I, Kalampalikis N. Qu'est-ce que les focus groups? Bulletin de psychologie. 2004;57(3):237-43.

14. Tong A, Sainsbury P, Craig J. Consolidated criteria for reporting qualitative research (COREQ) : a 32 -item checklist for interviews and focus groups. International Qual Health Care. 2007;19(6):349-57.

15. Lejeune C. Manuel d'analyse qualitative. Analyser sans compter ni classer. Paris : De Boeck Supérieur ; 2014. 149 p.

16. Ministère des affaires sociales, de la santé et des droits des femmes. Le pacte territoire santé : pour lutter contre les déserts médicaux. 2015 Disponible sur : <http://www.sante.gouv.fr/le-pacte-territoire-sante-pour-lutter-contre-les-deserts-medicaux,12793.html>.

17. Baschet-Vernet M. Colloque MG France : pour un virage ambulatoire cohérent et réussi ! 2015. Disponible sur : <http://www.mgfrance. org/index.php/actualite/politique-de-sante/812-pour-un -ambulatoire-coherent-et-reussi>.

18. Humanis. Résultats du Baromètre Santé Humanis 2013. 2013. Disponible sur : <https://presse.humanis.com/actualites/resultats-du-barometre-sante-humanis-2013-1ad7-186aa.html>.

19. Vinel V, Kessler-Bilthauer D, Volery I, Kivits J, Lamongie L, Chrisotphe S. Se soigner en zones rurales lorraines : trajectoires de soins, perceptions des territoires et obstacles à l'égalité de l'accès aux soins médicaux. Nancy (France) :Maison des Sciences de I'Homme de Lorraine ; 2016.

20. Observatoire France très haut débit. [internet]. 2016 [visitée le 3 janvier 2016]. Disponible sur :<http:/ / observatoire.francethd.fr>. 
21. Centre hospitalier régional universitaire de Nancy. Téléconsultations gériatriques : une première en Lorraine [Internet]. 2015. Disponible sur: <http://www.chu-nancy.fr/index.php/51-telemedecine>.

22. Agence régionale de santé de Lorraine. Lorraine : lancement réussi du service ODYS pour les premières téléconsultations en unité de dialyse médicalisée (UDM). 2013. Disponible sur : <www.ars.lorraine.sante.fr>.

23. Arnaud M. Téléconsultation en addictologie en Meuse : intérêts et reproductibilité à partir d'une expérimentation au sein du pôle de santé pluri-professionnel de Revigny. [Thèse de médecine générale]. [Nancy (France)] : Université de Lorraine ; 2015.

24. Baudier F, Bourgueil Y, Evrard I, Gautier A, Le Fur P, Mousquès J. La dynamique de regroupement des médecins généralistes libéraux de 1998 à 2009. Questions d'économie de la santé. 2010 ; (157). Disponible sur: <http://www.irdes.fr/Publications/2010/Qes157.pdf>.

25. Bidault Diallo A. Télémédecine et dossier médical personnel: perceptions et attentes des médecins généralistes. [Thèse de médecine générale]. [Angers France)] : Université d'Angers ; 2013.

26. Tesnière $A$, Leloup $P$, Quéreux $G$, Maillard $H$, Pedailles $S$, Leccia M-T, et al. Avis dermatologiques à distance: une enquête interrégionale. Annales de dermatologie et de vénéréologie. févr 2015;142(2):85-93.

27. Mines Paris. Évaluation d'un dispositif de télémédecine-TELEGERIA les nouvelles technologies au service des personnes âgées. [Internet]. 2007. Disponible sur : <http://docplayer.fr/1631482-Evaluation-dun-dispositif-de-telemedecine.html $>$.

28. Mathieu-Fritz A, Esterle L. Les transformations des pratiques professionnelles lors des téléconsultations médicales. Coopération interprofessionnelle et délégation des tâches. Revue française de sociologie. 2013;54(2):303-29.

29. Swinton JJ, Robinson WD, Bischoff RJ. Telehealth and rural depression: Physician and patient perspectives. Fam Syst Health. 2009;27(2):172-82.
30. Freidson E. La Profession médicale. Paris : Payot; 1984. 369 p.

31. République Française. Code de la sécurité sociale. Article L162-5-3. Disponible sur : <legifrance.gouv.fr>.

32. Troptard A-M. Étude des motifs de non-télétransmission des feuilles de soins par les médecins en France. [Thèse de médecine générale]. [Strasbourg (France)] : Faculté de Médecine de Strasbourg ; 2014. Disponible sur : <apima.org>.

33. Conseil national de l'ordre des médecins. Télémédecine, les préconisations du Conseil national de l'ordre des médecins. [Internet]. 2009. Disponible sur: <www.conseil-national.medecin.fr/sites/ default/files/telemedecine2009.pdf>.

34. Simon P. La place du téléconseil médical personnalisé dans les pratiques de télémédecine. European research in telemedicine/La Recherche européenne en Télémédecine. mars 2015; Disponible sur: <http://www.sciencedirect.com/science/article/pii/ S2212764X15000278>.

35. Lucas J. Télémédecine et autres prestations médicales électroniques. [Internet]. Ordre national des médecins; février 2016. Disponible sur : <www.conseil-national.medecin.fr/sites/default/files/uberisation_de_la_sante.pdf>.

36. Simon P, Acker D. La place de la télémédecine dans l'organisation des soins [Internet]. novembre 2008. Disponible sur : <www.sante. gouv.fr/IMG/pdf/Rapport_final_Telemedecine.pdf>.

37. Rédaction TICsanté. L'Antel lance une offre de formation en télémédecine. ticsante.com. 2014. Disponible sur : <http://www.ticsante. com/I-Antel-lance-une-offre-de-formation-en-telemedecineNS_1850.html>.

38. Télémédecine360. Liste des formations de télémédecine en France. 2015. Disponible sur : <www.telemedecine-360.com/wp-content/ uploads/Dossier_4_Formation.pdf>. 